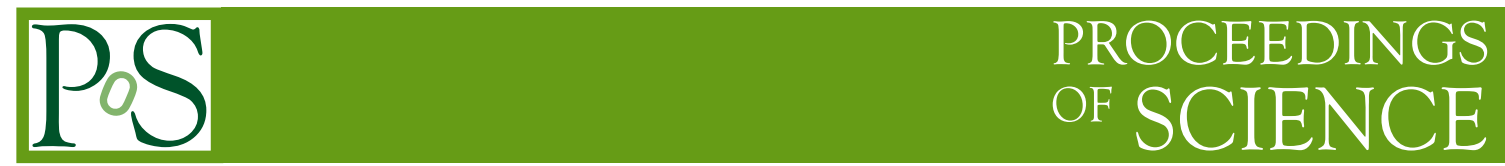

\title{
SM background estimation in the 1-lepton channel in SUSY searches (ATLAS)
}

\author{
Jeanette LORENZ* \\ on behalf of the ATLAS Collaboration \\ LMU Muenchen \\ E-mail: Jeanette.Lorenz@physik.uni-muenchen.de
}

\begin{abstract}
One of the promising channels in the search for Supersymmetry is the 1-lepton channel which requires one isolated electron or muon, multiple jets and a high missing transverse energy. Some SM processes like top, $\mathrm{W}$ and QCD can show similar experimental signatures. The magnitude of these backgrounds must be understood well. Various methods to estimate these backgrounds are presented.
\end{abstract}

The 2011 Europhysics Conference on High Energy Physics-HEP 2011,

July 21-27, 2011

Grenoble, Rhône-Alpes France

${ }^{*}$ Speaker. 

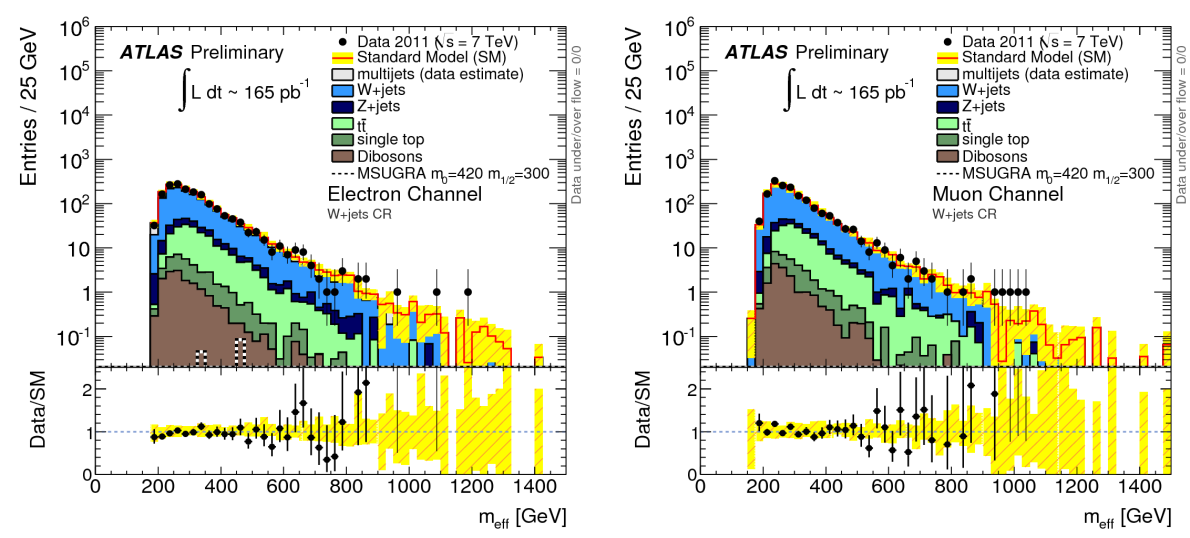

Figure 1: Distribution of $m_{\mathrm{eff}}$ in the $\mathrm{W}$ region for the electron and the muon channels. [1]

\section{Search for SUSY in final states with one lepton}

In $p-p$ collisions at a center-of-mass energy of $7 \mathrm{TeV}$ at the LHC, gluinos and squarks are expected to be produced copiously if they are light enough. Assuming a R-parity conserving SUSY model squarks and gluinos decay via a decay chain into the lightest supersymmetric particle, which is neutral, stable and weakly interacting. As a result, it escapes the ATLAS detector and is measured via missing transverse energy. This decay chain can also contain jets (from gluino and squark decays) and one or more leptons (from chargino and heavy neutralino decays). For this SUSY search [1] events with exactly 1 isolated lepton (electron with $p_{\mathrm{T}}>25 \mathrm{GeV}$ or muon with $p_{\mathrm{T}}>20 \mathrm{GeV}$ ),

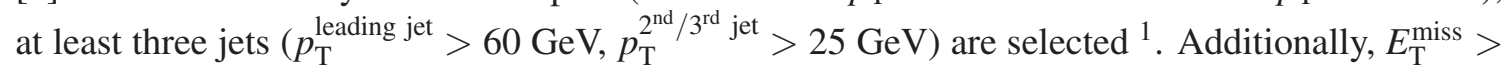
$125 \mathrm{GeV}, M_{\mathrm{T}}>100 \mathrm{GeV}^{2}$ and $m_{\mathrm{eff}}>500 \mathrm{GeV}^{3}$ are required. The two largest backgrounds after these cuts are $t \bar{t}$ and $W+$ jets events. The QCD multijet background, such as heavy flavor decays, photon conversions and misreconstructed jets, is heavily suppressed by the requirements of one isolated lepton and a large $E_{\mathrm{T}}^{\mathrm{miss}}$.

\section{Background estimation}

In order to estimate the $t \bar{t}$ and $W+$ jets backgrounds, the same signal selection cuts are applied as explained above, but using $30<E_{\mathrm{T}}^{\text {miss }}<80 \mathrm{GeV}$ and $40<M_{\mathrm{T}}<80 \mathrm{GeV}$. Two control regions using this selection are defined: $W+$ jets events (WR) where no b-tagged jet may be present in the three leading jets, and $t \bar{t}$ events (TR) where at least one b-tagged jet was found. The number of $t \bar{t}$ and $W+$ jets background events in the signal region are obtained by scaling the number of $t \bar{t}$ or $W+$ jets events in the control regions with a extrapolation factor which is obtained from Monte Carlo.

This method is validated by comparing Monte Carlo expectation and data in the control regions as shown in figure 1.

\footnotetext{
${ }^{1}$ the full list of all cuts applied can be found in [1]

${ }^{2}$ the transverse mass is defined as $m_{\mathrm{T}}=\sqrt{2 \cdot p_{\mathrm{T}}^{l} \cdot E_{\mathrm{T}}^{\text {miss }} \cdot\left(1-\cos \left(\Delta \phi\left(l, E_{\mathrm{T}}^{\text {miss }}\right)\right)\right)}$

${ }^{3}$ the effective mass is defined as $M_{\mathrm{eff}}=p_{\mathrm{T}}^{l}+E_{\mathrm{T}}^{\mathrm{miss}}+\sum_{i=1}^{3} p_{T}^{\mathrm{jet}_{\mathrm{i}}}$
} 

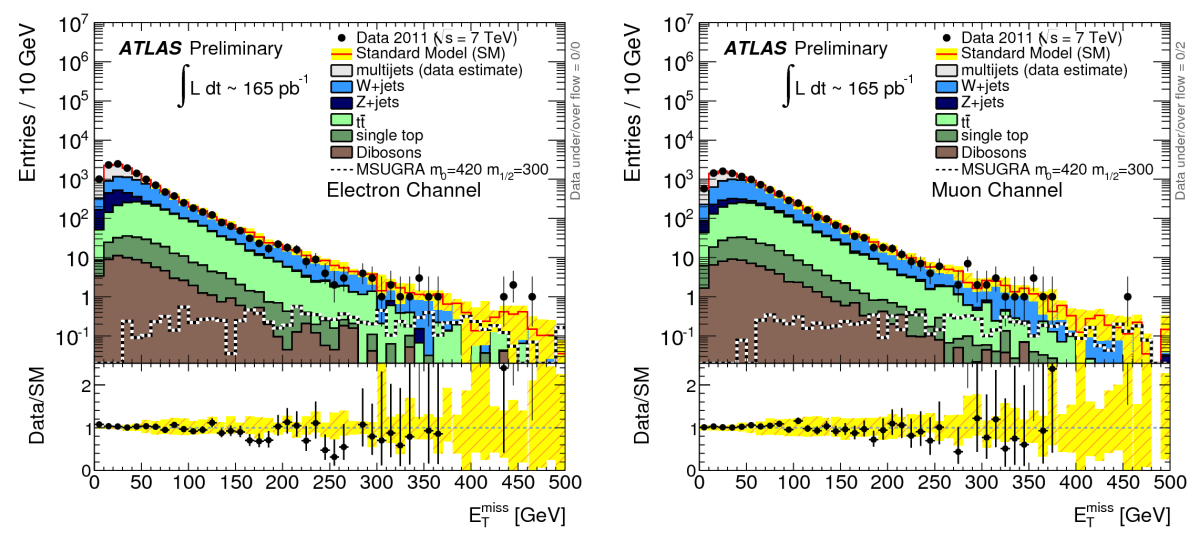

Figure 2: The $E_{\mathrm{T}}^{\text {miss }}$ distributions for the electron and the muon channels after applying only the lepton and jet cuts. [1]

The QCD multijet background is estimated by a matrix method. A loose control sample is defined by relaxing the identification criteria for the selected lepton in the signal selection cuts. The efficiency, $\varepsilon_{\text {fake }}$, for QCD multijet events to pass both the loose and the standard selection cuts is calculated in a control region enriched by QCD multijet events: this control region contains events with exactly one lepton, at least one jet (muons: $p_{\mathrm{T}}>60 \mathrm{GeV}$, electrons: $p_{\mathrm{T}}>30 \mathrm{GeV}$ ) and $E_{\mathrm{T}}^{\text {miss }}<30 \mathrm{GeV}$. Non-QCD multijet events in the control region are subtracted by using Monte Carlo. Similarly, an efficiency, $\varepsilon_{\text {real }}$, that non-QCD events pass both the loose and the standard selection cuts is estimated. In the muon channel $\varepsilon_{\text {real }}$ is calculated from $t \bar{t}$ and $W+$ jets Monte Carlo events, in the electron channel by a tag and probe method with Zee Monte Carlo events. All events passing the standard selection cuts can be expressed as sum of QCD multijet events (fake) and nonQCD events (real), $N_{\text {pass }}=\varepsilon_{\text {real }} N_{\text {real }}+\varepsilon_{\text {fake }} N_{\text {fake. }}$. Similarly for the events passing only the loose but failing the standard selection cuts, $N_{\text {fail }}=\left(1-\varepsilon_{\text {real }}\right) N_{\text {real }}+\left(1-\varepsilon_{\text {fake }}\right) N_{\text {fake }}$.

These two equations are solved for the number of QCD multijet events in the signal region:

$$
N_{\text {fake }}^{\text {pass }}=\varepsilon_{\text {fake }} N_{\text {fake }}=\frac{N_{\text {fail }}-\left(1 / \varepsilon_{\text {real }}-1\right) N_{\text {pass }}}{1 / \varepsilon_{\text {fake }}-1 / \varepsilon_{\text {real }}}
$$

Figure 2 shows the good agreement between the QCD multijet estimate and data.

\section{Results}

Contamination in the control regions from other backgrounds is taken into account by a combined fit to the control and signal regions. The total background in the signal region is $14.5 \pm 5.2$ events (electron channel) and $12.2 \pm 3.8$ events (muon channel), while 10 events (electron channel) and 12 events (muon channel) are observed (with an integrated luminosity of $165 \mathrm{pb}^{-1}$ ).

\section{References}

[1] The ATLAS Collaboration, Search for supersymmetry with jets, missing transverse momentum and one lepton at $\sqrt{s}=7 \mathrm{TeV}$, ATLAS-CONF-2011-090,

http: //cdsweb.cern.ch/record/1358623 